\title{
Finding the Shortest Move-Sequence in the Graph-Generalized 15-Puzzle is NP-Hard
}

\author{
Oded Goldreich
}

\begin{abstract}
Following Wilson (J. Comb. Th. (B), 1975), Johnson (J. of Alg., 1983), and Kornhauser, Miller and Spirakis (25th FOCS, 1984), we consider a game that consists of moving distinct pebbles along the edges of an undirected graph. At most one pebble may reside in each vertex at any time, and it is only allowed to move one pebble at a time (which means that the pebble must be moved to a previously empty vertex). We show that the problem of finding the shortest sequence of moves between two given "pebble configuations" is NP-Hard.
\end{abstract}

Keywords: NP-Completeness, Games' Complexity, Computational Group Theory

This work was completed in July 1984, and later appeared as Technical Report No. 792 of the Computer Science Department of the Technion (Israel). The current revision is quite minimal.

\section{Problem's Definition}

The following generalization of the "15-Puzzle" appeared in $[4,2,3]$ :

Board: The game is played on a finite, undirected, simple graph. The graph will be denoted by $G(V, E)$.

Legal Board Configuration: Every vertex contains at most one pebble, and one vertex is empty. That is, BC : $V \rightarrow\{0,1,2, \ldots,|V|-1\}$ is a legal board configuration if it is one-to-one and onto. The board configuration is interpreted as follows: if $\operatorname{BC}(v) \neq 0$, then vertex $v$ contains pebble $\operatorname{BC}(v)$, and if $\operatorname{BC}(v)=0$, then vertex $v$ is empty.

Legal Moves: A legal move consists of moving a single pebble, along one of the edges of the graph to an empty vertex. A legal move is a transformation on the set of legal configurations. Let $\mathrm{BC}(\cdot)$ be a legal configuration and $\mathrm{BC}^{\prime}(\cdot)$ be the configuration that results from $\mathrm{BC}(\cdot)$ after a legal move. Then, there exist two adjacent vertices, $u, v \in V$ (i.e., $(u, v) \in E$ ), such that $\mathrm{BC}^{\prime}(u)=\mathrm{BC}(v)$, $\mathrm{BC}^{\prime}(v)=\mathrm{BC}(u)=0$, and $\mathrm{BC}^{\prime}(w)=\mathrm{BC}(w)$ for all $w \in V \backslash\{u, v\}$. In this move the pebble $\mathrm{BC}(v)$ is moved from vertex $v$ to vertex $u$. 
A sequence of moves: A sequence of $t$ moves is a sequence of legal board configurations, denoted $\mathrm{BC}_{0}(\cdot), \mathrm{BC}_{1}(\cdot), \mathrm{BC}_{2}(\cdot), \ldots, \mathrm{BC}_{t}(\cdot)$, such that for $i=1, \ldots, t$ it holds that $\mathrm{BC}_{i}(\cdot)$ is the result of applying a legal move to $\mathrm{BC}_{i-1}(\cdot)$. The configuration $\mathrm{BC}_{0}(\cdot)$ is called the beginning configuration of the above sequence, and the configuration $\mathrm{BC}_{t}(\cdot)$ is called the finishing configuration of the above sequence.

Solutions: A pair of legal board configurations is said to have a solution if there exists a sequence of moves beginning at the first and finishing at the second.

\section{Prior Work}

Kornhauser, Miller and Spirakis [3] showed that, for any nonseparable graph $G(V, E)$, if a pair of legal board configurations has a solution, then it has a solution by $O\left(|V|^{3}\right)$ moves. Furthermore, they showed that such a solution (by $O\left(|V|^{3}\right)$ moves) can be found in $O\left(|V|^{3}\right)$ time. A natural algorithmic question arises:

Given a pair of legal board configurations that does have a solution, Is it feasible to find the shortest solution?

We answer this question negatively, proving that finding such a solution is NPHard.

\section{The NP-Completeness Result}

In order to discuss the problem of finding the shortest solution to a solvable pair of legal board configurations, we introduce the following decision problem, herafter referred to as the Shortest Move Sequence (SMS) Problem:

Input: A nonseparable, simple, undirected graph $G(V, E)$; a pair, $B(\cdot)$ and $F(\cdot)$, of legal board configuration; and an integer $K$.

Question: Is there a sequence of $K$ (or less) legal moves beginning at $B(\cdot)$ and finishing at $F(\cdot)$ ?

We prove the following result.

Theorem: The Shortest Move Sequence (SMS) problem is NP-Complete.

Proof: First note that SMS is in NP (since, w.l.o.g., $K=O\left(|V|^{3}\right)$ ). We prove that SMS is complete by reducing 3-Exact-Cover (3XC) to it. Recall that the $3 \mathrm{XC}$ is defined as follows:

Input: A set $U=\left\{e_{i}\right\}_{i=1}^{3 n}$ and a collection $S=\left\{s_{j}\right\}_{j=1}^{m}$ of 3-element subsets (3-subsets) of $U$.

Question: Is there a subcollection, $S^{\prime} \subseteq S$, such that every element in $U$ occurs in exactly one member of $S^{\prime}$ ?

If existing, such a collection, $S^{\prime}$, is called an exact cover. (Also, $|S|=n$.) 
Recall that Karp has proved that $3 \mathrm{XC}$ is NP-complete (see [1]). Now, given an instance of $3 \mathrm{XC}$, denoted $\left(U=\left\{e_{i}\right\}_{i=1}^{3 n}, S=\left\{s_{j}\right\}_{j=1}^{m}\right)$, we construct the following SMS instance:

- Let $V=U^{0} \cup U^{1} \cup S \cup\{t\}$, where $U^{\sigma}=\left\{e^{\sigma}: e \in U\right\}$ for $\sigma \in\{0,1\}$. The vertices $e^{0}$ and $e^{1}$ will be associated with the element $e \in U$. The vertices in $S$ will be associated with the corresponding 3 -subsets. The vertex $t$ will be called the temporary vertex.

- Let $E=E_{3 \mathrm{XC}} \cup\{(t, s): s \in S\} \cup\left\{\left(e^{0}, e^{1}\right): e \in U\right\}$, where

$$
E_{3 \mathrm{XC}}=\left\{\left(e^{\sigma}, s\right): \sigma \in\{0,1\} \wedge e \in U \wedge e \in s\right\}
$$

The edges in $E_{3 \mathrm{XC}}$ encode the description of the $3 \mathrm{XC}$ instance. Note that $\left(e^{\sigma}, s\right) \in E_{3 \mathrm{XC}}$ iff the element $e \in U$ appears in the 3-subset $s \in S$.

- Let $B\left(e_{i}^{\sigma}\right)=2 i-1+\sigma$, for $1 \leq i \leq 3 n$ and $\sigma \in\{0,1\}$, and $B\left(s_{j}\right)=6 n+j$, for $1 \leq j \leq m$. Let $B(t)=0$.

In the begin configurations $t$ is empty while the pebbles are placed in a "canonical" order. In particular, the pebbles $2 i-1$ and $2 i$, which are associated with the element $e_{i}$ (for $1 \leq i \leq 3 n$ ), are placed in vertices $e_{i}^{0}$ and $e_{i}^{1}$, respectively. The pebble $6 n+j$, which is associated with the 3 -subset $s_{j}$ (for $1 \leq j \leq m)$, is placed in vertex $s_{j}$.

- Let $F\left(e_{i}^{\sigma}\right)=2 i-\sigma$, for $1 \leq i \leq 3 n$ and $\sigma \in\{0,1\}$, and $F\left(s_{j}\right)=6 n+j$, for $1 \leq j \leq m$. Let $F(t)=0$.

In the finish configurations $t$ is still empty and the pebbles in the vertices that are associated with the 3 -subsets remain invariant w.r.t the begin configuration. The pebbles associated with each element of $U$ are switched w.r.t the begin configuration.

- Finally, let $K=11 n$.

Having presented our reduction it remains to show that it is indeed valid.

Assume that the $3 \mathrm{XC}$ instance has an exact cover, denoted $S^{\prime}=\left\{s_{i_{j}}\right\}_{j=1}^{n}$. Let $f:\{1,2, \ldots, n\} \times\{1,2,3\} \rightarrow\{1,2, \ldots, 3 n\}$ such that $e_{f(j, k)}$ is the $k$-th element in the 3 -subset $s_{i_{j}}$ (where the order on the elements in each 3 -subset is induced by an ordering of $U)$. Note that $s_{i_{j}}=\left\{e_{f(j, 1)}, e_{f(j, 2)}, e_{f(j, 3)}\right\}$ and $U=\left\{e_{f(j, k)}: 1 \leq j \leq n \wedge 1 \leq k \leq 3\right\}$. Then, following is a solution to the corresponding SMS instance:

$\underline{\text { for }} j=1$ to $n \underline{\text { do }} \underline{\text { begin }}$

move pebble $6 n+i_{j}$ from $s_{i_{j}}$ to $t$

$\underline{\text { for }} k=1$ to $3 \underline{\text { do }} \underline{\text { begin }}$

move pebble $\overline{2 f(j, k)}-1$ from $e_{f(j, k)}^{0}$ to $s_{i_{j}}$;

move pebble $2 f(j, k)$ from $e_{f(j, k)}^{1}$ to $e_{f(j, k)}^{0}$;

move pebble $2 f(j, k)-1$ from $s_{i_{j}}$ to $e_{f(j, k)}^{1}$;

[Comment: At this stage, for every $k \in\{1,2,3\}$,

the pebbles $2 f(j, k)-1$ and $2 f(j, k)$ are switched.]

$\underline{\text { end }}$

move pebble $6 n+i_{j}$ from $t$ to $s_{i_{j}}$; 
[Comment: At this stage all pebbles associated to elements in $s_{i_{j}}$ are switched and all the pebbles associated with 3 -subsets are back in place.] $\underline{\text { end }}$

One can easily verify that the foregoing procedure transforms the begin configuration into the finish configuration in $(1+3 \cdot 3+1) \cdot n=K$ moves

Assume, on the other hand, that the SMS instance has a solution in no more than $K=11 n$ moves. Let us denote this solution (i.e., sequence of moves) by $Q$. Recall that in each move a single pebble is moved (to an empty vertex). The following facts concerning $Q$ can be easily verified:

Fact 1: Switching pebble $2 i-1$ with pebble $2 i(1 \leq i \leq 3 n)$ requires at least two moves of one of these pebbles and at least one move of the other pebble. Furthermore, this switching requires that at least one of these pebbles passes through a vertex associated with a 3 -subset that contains the element $e_{i}$.

The main part follows from the fact that each move must be to a previously empty vertex, and the furthermore part follows by the graph's structure.

Fact 2: If some pebble passes through a 3 -subset vertex $s_{j}(1 \leq j \leq m)$ during $Q$, then the pebble $6 n+j$ must have been moved during $Q$.

Let $M$ denote the set of pebbles that are associated with 3-subsets that moved during $Q$. Using Facts 1 and 2, we get.

Fact 3: The number of moves in $Q$ is at least $3 \cdot 3 n+2 \cdot|M|$.

Recall the number of moves (in $Q$ ) is at most $K=11 n$. Thus:

Fact 4: $|M| \leq n$.

Fact 5: The collection $C=\left\{s_{j}: 6 n+j \in M\right\}$ constitutes a cover of the set $U$. That is, for every element $e \in U$, there exists a 3 -subset $s \in C$ such that $e \in s$.

Note that $2 i-1$ has been switched with $2 i$, for each $1 \leq i \leq 3 n$, and by Facts 1 and 2 this implies that for some $j$ such that $e_{i} \in s_{j}$ it holds that $6 n+j \in M$.

Combining Facts 4 and 5, we conclude that $C$ is an exact cover of the $3 \mathrm{XC}$ instance. This completes the proof of the theorem.

\section{Acknowledgements}

I am grateful to Shimon Even, Dan Kornhauser, Silvio Micali and Gary Miller for very helpful discussions. 


\section{References}

1. M.R. Garey and D.S. Johnson: Computers and Intractability: A Guide to the Theory of NP-Completeness, page 221. Freeman, San Francisco, 1979.

2. D.S. Johnson: The NP-Completeness Column: An Ongoing Guide, J. of Algorithms, 4, pp. 397-411, 1983.

3. D.M. Kornhauser, G. Miller and P. Spirakis: Coordinating Pebble Motion on Graphs, the Diameter of Permutation Groups, and Applications. In Proc. of the 25th FOCS, pages 241-250, 1984.

4. R.W. Wilson: Graphs, Puzzles, Homotopy, and Alternating Groups. J. of Comb. Th. (B), 16, pages 86-96, 1974. 